\title{
Phosphorylation and function of DGAT1 in skeletal muscle cells
}

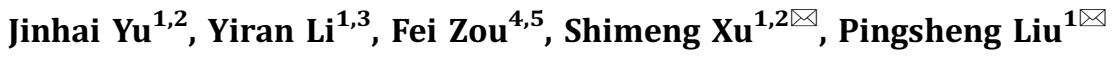 \\ ${ }^{1}$ National Laboratory of Biomacromolecules, Institute of Biophysics, Chinese Academy of Sciences, Beijing 100101, \\ China \\ ${ }^{2}$ University of Chinese Academy of Sciences, Beijing 100049, China \\ 3 Department of Biological Science and Biotechnology, School of Biological Science and Medical Engineering, Beihang \\ University, Beijing 100191, China \\ ${ }^{4}$ Graduate School of Anhui Medical University, Hefei 230032, China \\ ${ }^{5}$ General Hospital of Air Force, Beijing 100142, China
}

Received: 8 January 2015 / Accepted: 27 April 2015 / Published online: 21 August 2015

\begin{abstract}
Aberrant intramuscular triacylglycerol (TAG) storage in human skeletal muscle is closely related to insulin insensitivity. Excessive lipid storage can induce insulin resistance of skeletal muscle, and under severe conditions, lead to type 2 diabetes. The balance of interconversion between diacylglycerol and TAG greatly influences lipid storage and utilization. Diacylglycerol 0-acyltransferase 1 (DGAT1) plays a key role in this process, but its activation and phosphorylation requires further dissection. In this study, 12 putative conserved phosphorylation sites of DGAT1 were identified by examining amino acid conservation of DGAT1 in different species. Another 12 putative phosphorylation sites were also found based on bioinformatics predictions and previous reports. Meanwhile, several phosphorylation sites of DGAT1 were verified by phosphorylation mass spectrometry analysis of purified DGAT1 from mouse myoblast C2C12 cells. Using single point mutations, a regulatory role of 3 putative phosphorylation sites was dissected. Finally, using truncation mutations, a potential domain of DGAT1 that was involved in the regulation of enzymatic activity was revealed. This study provides useful information for further understanding DGAT1 activity regulation.
\end{abstract}

Keywords DGAT1, Phosphorylation, Single point mutation, Enzymatic activity

\section{INTRODUCTION}

Triacylglycerol (TAG) is the main energy storage compound in mammals. Due to their hydrophobicity, TAG has an energy density six times greater than hydrated glycogen (Yen et al. 2008). TAG is the main component of plant seed oils, which are important for the human diet as well as in industrial applications. Furthermore,

$\triangle$ Correspondence: smxu@moon.ibp.ac.cn (S. Xu), pliu@ibp.ac.cn (P. Liu)
TAG from plants and microorganisms can be transformed to biofuels.

In mammals, TAG is mainly stored in adipocytes but also stored in mammary epithelial cells, hepatocytes, enterocytes, and myocytes. TAG synthesized and stored in hepatocytes and enterocytes are used primarily for lipoprotein assembly and secretion. In myocytes, TAG serves as energy storage, where they are hydrolyzed to free fatty acids (FFAs) to provide substrates for mitochondrial $\beta$-oxidation and the production of adenosine triphosphate (ATP).

However, ectopic storage of TAG is closely related to human diseases, such as obesity, diabetes, and 
steatohepatitis. Insulin-regulated glucose metabolism is mainly accomplished by skeletal muscle in humans (Shulman et al. 1990), and insulin resistance (IR) in skeletal muscle is a main cause of type 2 diabetes (T2D). There is substantial evidence, suggesting that ectopic storage of TAG in myocytes is intimately related to IR (Kelley and Goodpaster 2001; Kelley et al. 2002; Machann et al. 2004; Moro et al. 2008; Perseghin et al. 1999). For example, knock out of acyl-CoA:diacylglycerol acyltransferase 1 (DGAT1) in mice effectively inhibited TAG accumulation induced by high fat diet and also alleviated IR (Chen et al. 2002). In another study, DGAT1 knockout increased insulin sensitivity of skeletal muscle and white fat tissue in mice fed a standard diet (Chen et al. 2004). However, it has been observed that the skeletal muscle of endurance athletes contains high levels of TAG storage but is acutely sensitive to insulin (Goodpaster et al. 2001; Russell et al. 2003). Similarly, another study found that acute exercise increased TAG synthesis while suppressing IR (Schenk and Horowitz 2007).

Although the precise mechanisms linking TAG storage in skeletal muscle and insulin sensitivity are not known, enzymes that synthesize TAG from diacylglycerol (DAG) and acetyl-CoA charged fatty acids are most likely involved in this process. Dgat1 and Dgat2 are the two main genes encoding the enzymes for this process (Cases et al. 1998, 2001). Although they catalyze the same reaction, the two enzymes belong to different families, have little sequence similarity, and have distinct tissue distributions. DGAT1 plays a major role in TAG synthesis skeletal muscle (unpublished data).

In most species, Dgat1 encodes a protein of $\sim 500$ amino acids with molecular weight $\sim 55 \mathrm{kDa}$. DGAT1 contains a large hydrophobic domain, localizes in the endoplasmic reticulum (ER), can form homo-tetramers through N-terminal interactions (Yen et al. 2008), and has a potential DAG binding site close to the $\mathrm{C}$ terminus. DGAT1 is regulated at both transcriptional and posttranscriptional levels. The protein is predicated to contain several sites which could be phosphorylated by protein kinase A (PKA) and protein kinase C (PKC) (Yen et al. 2008). The activity of DGAT1 is regulated by phosphorylation in rat livers and adipocytes (Haagsman et al. 1981, 1982). However, human DGAT1 with a Y316H mutation overexpressed in adipocytes did not show any apparent upregulation in enzymatic activity in an in vitro assay (Yu et al. 2002). DGAT1 was shown to be regulated post-transcriptionally by observing 3T3-L1 cells; during differentiation, mRNA level of DGAT1 was not proportional to its enzymatic activity (Cases et al. 1998; Yu et al. 2002). In this study, we investigated more potential phosphorylation sites of DGAT1. We assayed the enzymatic activities of proteins carrying different mutations at these sites, providing more clues for screening drugs regulating the activity of DGAT1. We also investigated the functional domains of DGAT1 by truncating it into different fragments to delineate the mechanisms regulating DGAT1 activity.

\section{RESULTS AND DISCUSSION}

\section{DGAT1 potential phosphorylation sites predication by informatics}

We compared DGAT1 amino acid sequences from 13 different species and found that DGAT1 was highly conserved from lower animals (e.g., C. elegans) to higher animals (e.g., human) (Fig. 1A). There were 12 potential phosphorylation sites (serine, threonine, and tyrosine) conserved across all species analyzed (Fig. 1B, red fonts indicated sites): S83, S86, S219, Y265, T271, Y274, S318, Y350, Y372, Y401, S422, and Y483 (sites in mouse DGAT1). There were another 12 potential phosphorylation sites (Fig. 1B, black fonts indicated sites) predicated by bioinformatics strategies (NetPhosK and PhosphoSitePlus) and previous literature: S9, S10, T15, S17, S20, S25, S40, S66, S67, S89, S244, and Y327 (Humphrey et al. 2013; Yu et al. 2002). Among these potential phosphorylation sites, only Y327 (human Y316) was confirmed as a phosphorylation site by mutagenesis (Yu et al. 2002), and T15 (Han et al. 2010), S17 (Huttlin et al. 2010; Kettenbach et al. 2011; Wilson-Grady et al, 2013), S20 (Han et al. 2010; Huttlin et al. 2010; Kettenbach et al. 2011; Villen et al. 2007; Wilson-Grady et al. 2013), S40 (Trinidad et al. 2012), and S67 (Huttlin et al. 2010; Wilson-Grady et al. 2013) were previously confirmed by phosphorylation mass spectrometry.

\section{DGAT1 protein enrichment and phosphorylation mass spectroscopy}

To confirm the phosphorylation of potential sites predicated by bioinformatic methods and to identify novel phosphorylation sites, we analyzed the enriched DGAT1 by phosphorylation mass spectrometry. We overexpressed Myc-His tagged mouse DGAT1 in C2C12, 
A
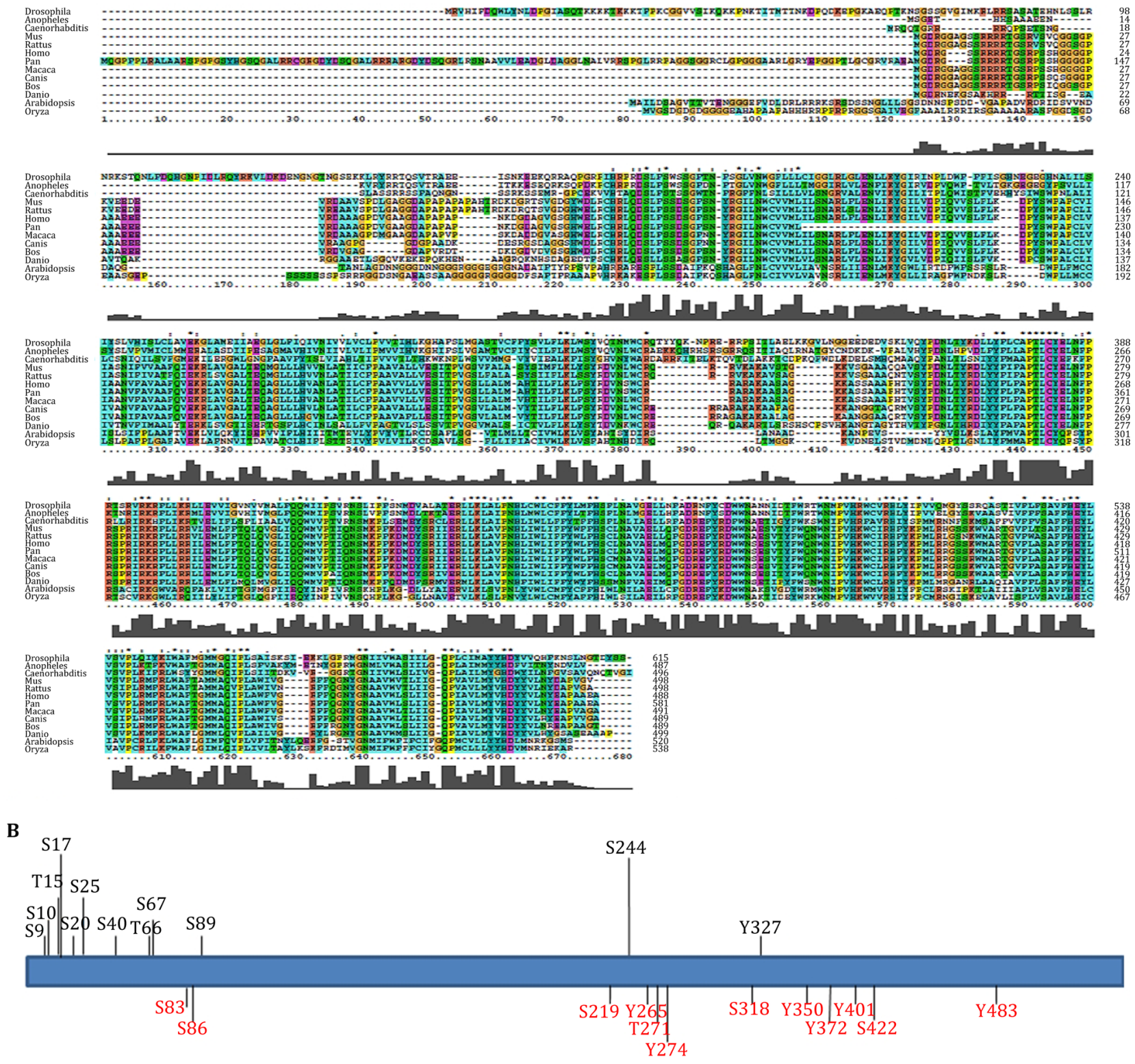

Fig. 1 The characteristics of DGAT1 amino acid sequence. A DGAT1 amino acid sequence was conserved from $C$. elegans to Human. DGAT1 from selected species were from NCBI and homology alignment was analyzed by ClustalX2. B Putative phosphorylation sites of DGAT1 (serine, threonine, and tyrosine). Red font indicated sites were conserved sites; black font indicated sites were potential sites predicted by bioinformatics or preliminarily identified in previous studies

enriched the protein by nickel-affinity chromatography, and confirmed with immunoblots. The DGAT1 was eluted with $500 \mathrm{mmol} / \mathrm{L}$ imidazole with no detectable cytosolic protein contamination. We further concentrated DGAT1 with ultra-filtration (Fig. 2A). Concentrated DGAT1 was separated by SDS-PAGE and visualized by Colloidal blue staining (Fig. 2B). The band containing DGAT1 was excised for phosphorylation mass spectrometry analysis. Peptides detected by mass spectrometry covered $1 / 4$ to $1 / 3$ of the sequence of DGAT1 and detected 6 phosphorylation sites: T15, S17, S20, S25, S40, and T66. The T66 site is a novel discovery, and the other sites have been reported previously, suggesting that our methods are efficient and could be used to detect further DGAT1 phosphorylation sites under different stimulation conditions. 

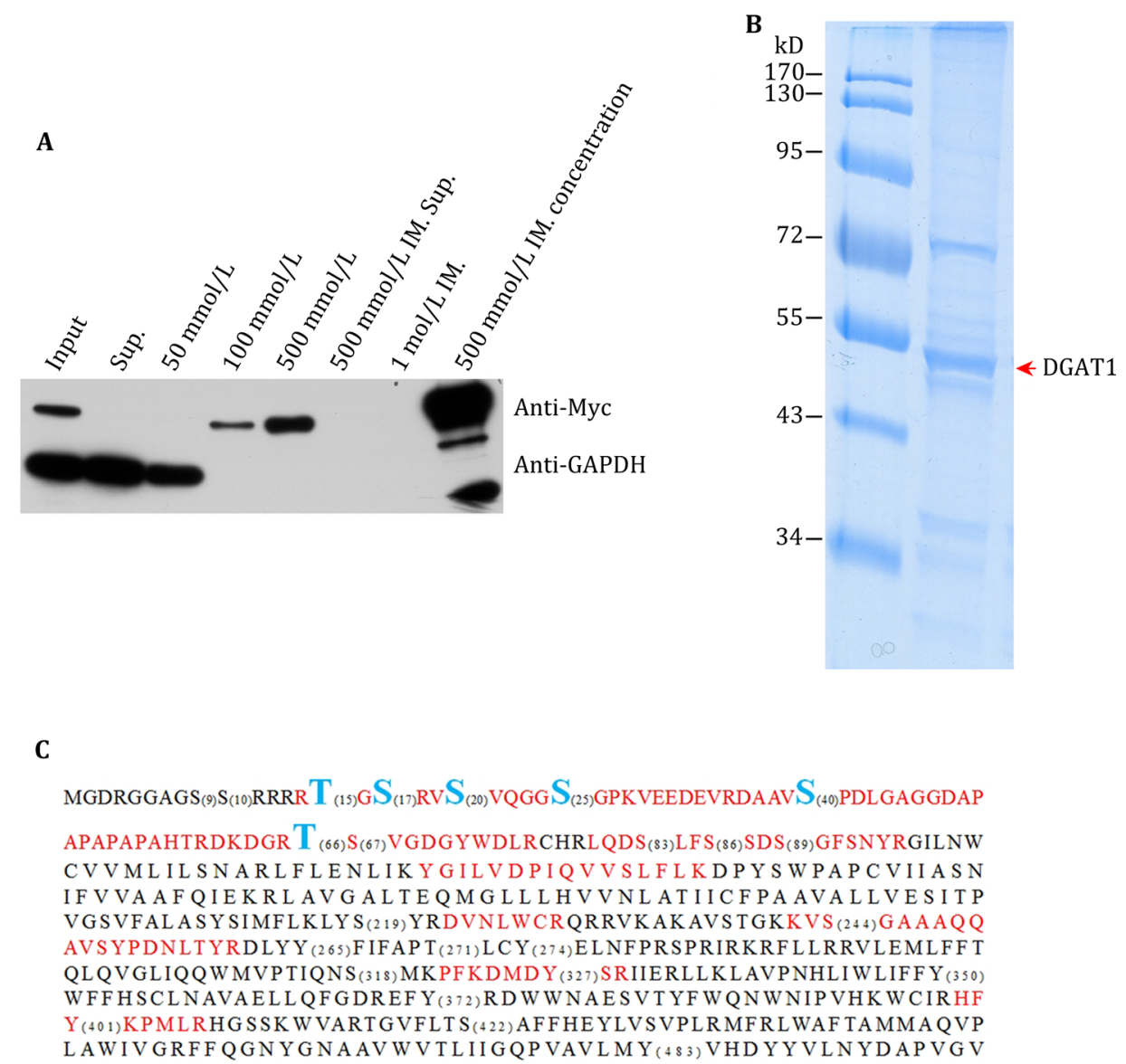

Fig. 2 Identification of DGAT1 phosphorylation sites by phosphorylation mass spectrometry. A DGAT1-His was overexpressed in C2C12 cells and purified by Ni-affinity chromatography. $500 \mathrm{mmol} / \mathrm{L}$ imidazole (IM) eluted sample was concentrated for further analysis. Sup: supernatant. B Enriched DGAT1 was analyzed by SDS-PAGE and Colloidal blue staining; red arrow indicated enriched DGAT1-His. C Phosphorylation mass spectrometry of enriched DGAT1, blue fonts indicated detected phosphorylation sites, red fonts indicated detected amino acid fragments, and numbers in parenthesis indicated putative phosphorylation sites

\section{DGAT1 single mutagenesis and enzymatic activity assay}

We mutated each of the 24 potential phosphorylation sites of DGAT1 one by one to either alanine (A) or glutamate (E) to mimic dephosphorylation or phosphorylation, respectively. These mutations showed different phenotypes when overexpressed in C2C12 cells (data not shown). However, only mutations of S83, S86, and S89 could apparently regulate DGAT1 enzymatic activity (Fig. 3). Overexpression of DGAT1-S83A in C2C12 cells resulted in a lower TAG/DAG ratio compared with overexpression of wild-type DGAT1 (DGAT1wt). Overexpression of DGAT1-S83E increased TAG/DAG ratio significantly compared with DGAT1-S83A overexpression (Fig. 3Aa). This ratio alteration suggested that the phosphorylation state of S83 might regulate DGAT1 activity.

We next compared the amount of DAG and TAG in wild type and cells overexpressing these three constructs. Although there was an increased quantity of TAG in cells overexpressing DGAT1-wt or DGAT1-S83E, the DAG content were unchanged from the control (Fig. 3Ab). This indicated that phosphorylation of S83 might also be related to the regulation of lipid uptake or DAG synthesis. One point must to be noted that the DGAT1-S83E protein was expressed much less than the other two DGAT1 proteins (Fig. 3Ac). 

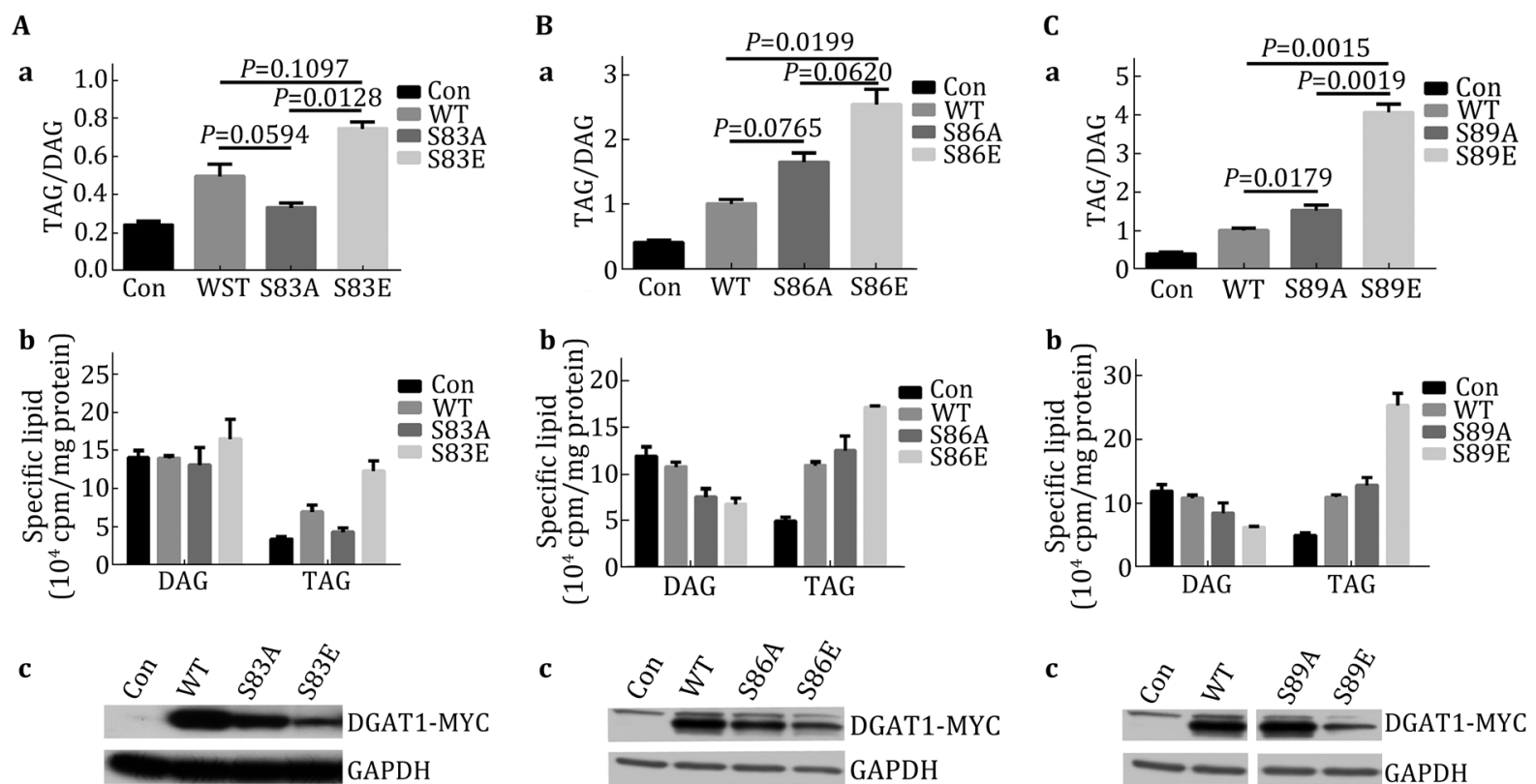

Fig. 3 Mutagenesis of DGAT1 putative phosphorylation sites and TAG synthetic activities. A Mutations of DGAT1 serine 83 and their enzymatic activities, (a) TAG/DAG ratio of vector, DGAT1-wt, S83A, and S83E overexpressed C2C12 cells, respectively; S83A and S83E showed significant differences of enzymatic activities, (b) specific lipid contents of different transfections, (c) DGAT1-Myc/His expression level of different transfections. B Mutations of serine 86 and their enzymatic activities, transfection with S86E significantly increases TAG/ DAG ratio compared with WT transfection. C Mutations of serine 89 and their enzymatic activities. S89E can significantly elevate DGAT enzymatic activity compared with WT and S89A. Data presented were analyzed by one-way ANOVA

DGAT1-S86A overexpression did not significantly alter the TAG/DAG ratio, compared with DGAT1-wt overexpression, while DGAT1-S86E overexpression dramatically and significantly increased the TAG/DAG ratio (Fig. 3Ba). In contrast with the S83 mutations, the increase amount of TAG as a result of the overexpression of S86E mutant was accompanied by a decrease of DAG (Fig. 3Bb). Similar to the S86E, but more strikingly and significant, overexpression of DGAT1-S89E drove TAG/DAG ratio up to more than four fold (Fig. 3Bc). However, neither DGAT1-S86A nor DGAT1-S89A decreased the TAG/DAG ratio or total amount of TAGs compared to DGAT1-wt overexpression. Collectively, these data suggested that the role of these two sites in regulating enzymatic activity was much more complex and may have other regulatory input besides phosphorylation regulation.

\section{$\mathrm{N}$-terminal of DGAT1 regulated its activity}

The point mutations of DGAT1 suggested that potential phosphorylation sites between S83 and S89 were important for regulation of DGAT enzymatic activity, and the $\mathrm{N}$-terminus was assumed to have a role in oligomer formation and acyl-CoA binding (McFie et al. 2010; Siloto et al. 2008; Weselake et al. 2006). To examine the role of the N-terminus of DGAT1, we prepared two mouse DGAT1 truncation mutants including a fragment consisting of amino acids (aa) 1-95 and the other consisting of aa 95-498 according to the ClustalX2 result. Overexpression of the 1-95 fragment of DGAT1 did not alter cell TAG/DAG ratio, while overexpression of the 95-498 fragment increased the ratio of TAG/DAG compared to overexpression of DGAT1-wt (Fig. 4A). These data suggest that the $\mathrm{N}$-terminus has no enzymatic activity but may negatively regulate DGAT1 activity. The total amount of TAG in cells overexpressing the 95-498 fragment was not different from cells overexpressing DGAT1-wt. However, these results may be due to the low expression level of the 95-498 fragment (Fig. 4B, C). To investigate further, we constructed another three truncation mutants randomly with about 20 aa interval: 118-498, 138-498, and 158-498. The three fragments had no apparent DGAT activity compared with 
A

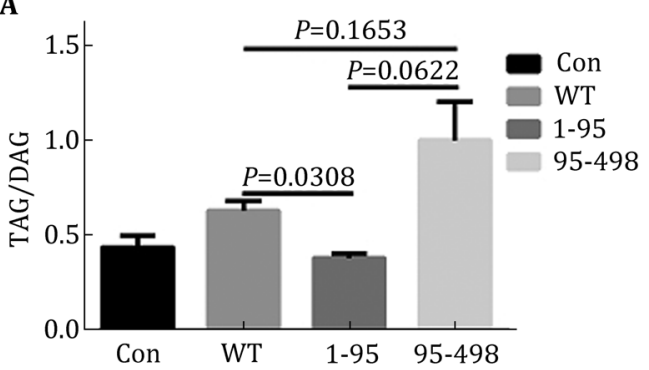

B

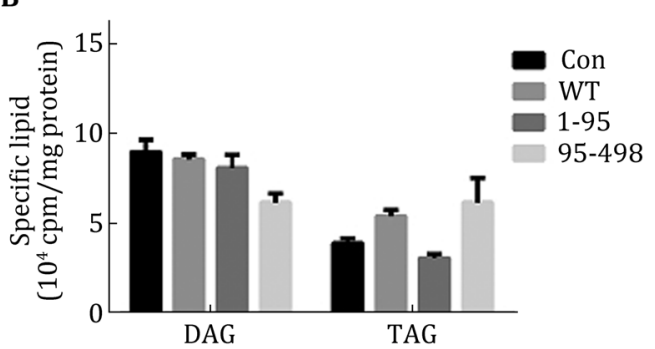

C

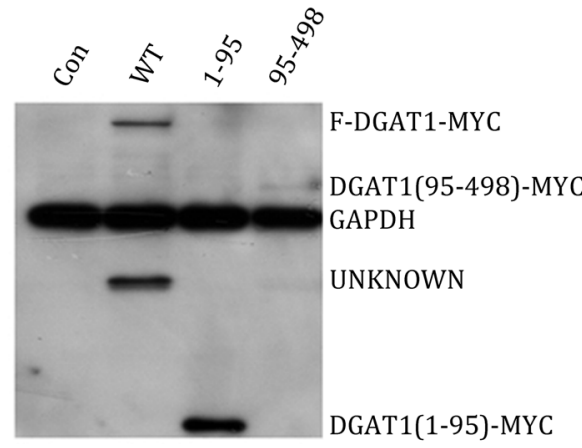

Fig. 4 N-terminus of DGAT1 repressed its enzymatic activity. A TAG/DAG ratio of different transfections of DGAT1 truncation mutants in C2C12, DGAT $1^{95-498}$ had higher enzymatic activity than WT, while DGAT $1^{1-95}$ had no enzymatic activity compared to vector. B Specific lipid composition of different transfections of DGAT truncation mutants in C2C12. C DGAT1 expression levels of different truncation mutations detected by Myc antibody, the expression level of DGAT1 ${ }^{95-498}$ was much lower than WT and DGAT1 $1^{1-95}$

DGAT1-wt and DGAT1-95-498 (Fig. 5), which suggested that aa 95-118 contained an important functional domain.

\section{CONCLUSION}

The overall prevalence of diabetes and prediabetes in the Chinese adult population were estimated in 2010 to be $11.6 \%$ and $50.1 \%$, respectively (Xu et al. 2013).
Skeletal muscle accounts for the majority of glucose consumption in humans (Shulman et al. 1990), and plays a critical role in maintaining glucose homeostasis. The ectopic storage of lipids has been identified as a major cause of diabetes (Samuel and Shulman 2012), and the excess storage of lipids in skeletal muscle significantly influences its insulin sensitivity. Recently, TAG, DAG, and other bioactive lipids were proposed to be the major cause of IR in skeletal muscle (Erion and Shulman 2010). The upregulation of myocellular DGAT1 has been demonstrated to augment TAG storage, decrease cellular DAG, and protect against high fat diet induced IR (Liu et al. 2007). The activity of DGAT1 was proposed to be regulated by post-translational modification (Yu et al. 2002) and PKA might be the upstream regulator (Haagsman et al. 1981, 1982; Rodriguez et al. 1992). Several potential phosphorylation sites have been studied previously (Han 2011; Humphrey et al. 2013), but none was found to regulate DGAT1 activity by biochemical methods.

Using single point mutagenesis, we found that conserved potential phosphorylation sites (S83, S86, and S89) in the N-terminus could have a great influence on the activity of DGAT1 in C2C12 cells. When each of these three serines was mutated to glutamate to mimic phosphorylation, there was an apparent increase in DGAT1 activity, suggested that phosphorylation at each of these three sites is likely to potentiate DGAT1 activity. However, we failed to detect phosphorylation at any of these three sites by phosphorylation mass spectrometry. The biochemically enriched DGAT1 might be at basal level when lacking upstream stimulating.

Truncation of the N-terminus of DGAT1 increased enzymatic activity, suggesting that $\mathrm{N}$-terminus possibly acted as a negative regulator, and the mechanisms underlying this phenomenon require further study. It is possible that the $\mathrm{N}$-terminus is required to permit the formation of homo-tetramers, which may suppress enzymatic activity (McFie et al. 2010). The DGAT1 truncation mutant lacking amino acids 95-118 demonstrated that this region was important for its enzymatic activity, which suggested that this domain might contain binding sites for acyl-CoA or DAG, or other essential element to fulfill the enzymatic function. Further study of the regulatory mechanisms of DGAT1 phosphorylation would provide valuable information for screening and developing drugs to lower ectopic TAG storage in non-adipocyte tissue, with implications for diabetes, obesity, and related metabolic disorders. 

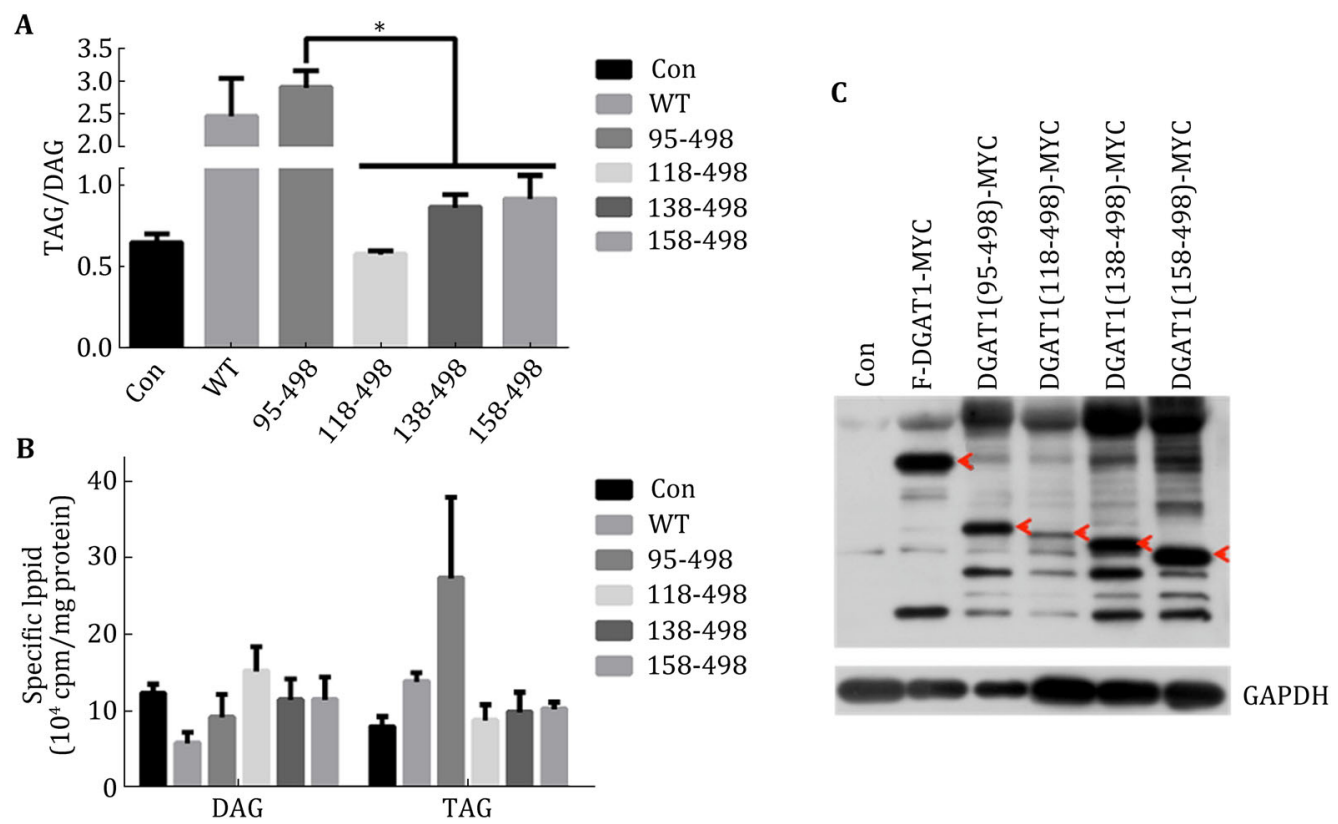

Fig. 5 The 95-118 amino acid fragment of DGAT1 was essential to enzymatic activity. A TAG/DAG ratio of different transfections of DGAT1 truncations in C2C12, DGAT1 ${ }^{95-498}$ had higher enzymatic activity than DGAT1 $1^{118-498}$, DGAT1 $1^{138-498}$, and DGAT1 ${ }^{158-498}$. B Lipid distribution of different transfections of DGAT truncation mutants in C2C12, the TAG accumulation of DGAT1 $1^{95-498}$ was greater than the other truncation mutants. C DGAT1 expression levels of different truncation mutants detected by Myc antibody

\section{MATERIALS AND METHODS}

\section{Materials}

The colloidal blue staining Kit, reverse transcriptase, and pcDNA3.1(+)/myc-his A plasmid were from Invitrogen. Sodium palmitate and the TAG mixed lipid standard were from Sigma-Aldrich. Tritium labeled palmitic acid was from PerkinElmer. Myc antibody was from CST. Antibody recognized GAPDH was from Millipore. The goat anti-rabbit secondary antibody and goat antimouse secondary antibody were from ZSGB-BIO.

\section{C2C12 cell culture}

Mouse C2C12 myoblasts (ATCC) were maintained in DMEM (Macgene Biotech.) supplemented with 10\% FBS (Hyclone), $100 \mathrm{U} / \mathrm{mL}$ penicillin, and $100 \mathrm{mg} / \mathrm{mL}$ streptomycin (Macgene Biotech.) at $37{ }^{\circ} \mathrm{C}, 5 \% \mathrm{CO}_{2}$. For plasmid transfection, $\mathrm{C} 2 \mathrm{C} 12$ cells were trypsinized, mixed with the indicated plasmids, and nucleoporated by a standard program according to instructions from Amaxa. The transfected cells were then seeded to 12-well plates and treated cells with FFAs as described previously (Peng et al. 2011).

\section{Plasmid constructions}

Cloning and mutagenesis primers are listed in Table 1. RNA extraction from C2C12 cells was conducted by using Trizol according to the manufacturer's protocol (Invitrogen). All plasmids used for transfection and further experiments were proved by sequencing.

\section{FFAs treatment and lipids extraction}

After C2C12 cells were transfected with the indicated plasmids and cultured for $24 \mathrm{~h}$, the cells were treated with FFAs. Complete medium was pre-warmed to $60{ }^{\circ} \mathrm{C}$ and sodium palmitate was added to a final concentration of $500 \mu \mathrm{mol} / \mathrm{L}$ with $5 \mu \mathrm{Ci} / \mathrm{mL}^{3} \mathrm{H}$-palmitic acid. The medium was cooled to $37{ }^{\circ} \mathrm{C}$, and cells were treated for $12 \mathrm{~h}$. After FFAs treatment, cells were washed three times with PBS and then lysed with $1 \%$ Triton X-100 (in PBS) at room temperature for $20 \mathrm{~min}$. Cell lysates were further processed with sonication and the protein concentration determined by BCA assay (Thermo). Lipids were extracted from the cell lysates by adding $1 \mathrm{~mL}$ lipid extraction buffer (chloroform:methanol:acetic acid $=50: 50: 1, v / v / v$ ). The mixture was vortexed and then centrifuged at $20,000 \mathrm{~g}$ for $10 \mathrm{~min}$ at $4{ }^{\circ} \mathrm{C}$. The 
Table 1 Primers sequences

\begin{tabular}{|c|c|c|}
\hline Primers & Forward & Reverse \\
\hline pcDNA3.1-dgatl & $\begin{array}{l}\text { 5'-GAGAATTCGCCACCATGGGCGACCGCGGAGG } \\
\text { CGCGGGAAGCTCTCG-3' }\end{array}$ & $\begin{array}{l}\text { 5'-AACTCGAGGACCCCCACTGGGGCATCG } \\
\text { TAGTTGAG-3' }\end{array}$ \\
\hline S83A & 5'-CATCGTCTGCAAGATGCTTTGTTCAG-3' & 5'-CATCTTGCAGACGATGGCACCTCAG-3' \\
\hline SS3E & 5'-ATCGTCTGCAAGATGAATTGTTCAGA-3' & 5'-TTCATCTTGCAGACGATGGCACCTCAGAT-3' \\
\hline S86A & 5'-CAAGATTCTTTGTTCGCCTCAGACAGT-3' & 5'-GCGAACAAAGAATCTTGCAGACGATG-3' \\
\hline S86E & 5'-AAGATTCTTTGTTCGAATCAGACAGT-3' & S'-TTCGAACAAAGAATCTTGCAGACGAT-3' \\
\hline S89A & 5'-TTGTTCAGCTCAGACGCTGGTTTCAGC-3' & 5'-GCGTCTGAGCTGAACAAAGAATCTTG-3' \\
\hline S89E & 5'-TTTGTTCAGCTCAGACGAAGGTTTCAGCA-3' & 5'-TTCGTCTGAGCTGAACAAAGAATCTTGCA-3' \\
\hline dgatl (1-95) & 5'-ATGAATTCGCCACCATGGGCGACCGCGGAGGCGC-3' & 5'-ATCTCGAGACGATAATTGCTGAAACCAC-3' \\
\hline dgatl (95-498) & 5'-ATGAATTCGCCACCATGCGTGGTATCCTGAATTGGTG-3' & 5'-ATCTCGAGGACCCCCACTGGGGCATCGT-3' \\
\hline dgatl (118-498) & 5'-ATGAATTCGCCACCATGATCAAGTATGGCATCCTGGT-3' & 5'-ATCTCGAGGACCCCCACTGGGGCATCGT-3' \\
\hline dgatl (138-498) & 5'ATGAATTCGCCACCATGTACAGCTGGCaGCCCCATG-3' & 5'-ATCCGAGGACCCCCACTGGGGCATCGT-3' \\
\hline dgatl (158-498) & 5'-ATGAATTCGCCACCATGCAGATTGAGAAGCGCCTGGCAGTGG-3' & 5'-ATCTCGAGGACCCCCACTGGGGCATCGT-3' \\
\hline
\end{tabular}

bottom lipid phase was transferred to fresh tubes for further analysis.

\section{Thin layer chromatography (TLC) and isotopic tracer assay}

The extracted lipids (30 $\mu \mathrm{L})$ were spotted onto TLC plates (Yantai Jiangyou silicone company) and developed with neutral lipids developing buffer (n-hexane: ether:acetic acid $=80: 20: 1, v / v / v)$. The lipids were visualized by iodine staining. The TAG and DAG fractions, identified relative to standards, were scraped into tubes. The lipids were quantified by liquid scintillation.

\section{DGAT1 protein enrichment and phosphorylation mass spectrometry}

DGAT1-Myc-His A overexpressed C2C12 cells were harvested in lysis buffer with $5 \mathrm{mmol} / \mathrm{L} \beta$-glycerophosphoric acid, $10 \mathrm{mmol} / \mathrm{L} \mathrm{NaF}, 1 \mathrm{mmol} / \mathrm{L} \mathrm{Na}_{3} \mathrm{VO}_{4}$, $10 \mathrm{mmol} / \mathrm{L}$ sodium pyrophosphate, and protease inhibitor cocktail and disrupted by sonication on ice. DGAT1 protein was enriched by Nickel-affinity chromatography (GE Health) and eluted with a step gradient of imidazole at $4{ }^{\circ} \mathrm{C}$. The eluate was concentrated by ultra-filtration (Millipore, $30 \mathrm{kDa}$ ). The chromatography was followed by immunoblots. The concentrated DGAT1 proteins were separated and visualized by SDS-PAGE and Colloidal blue staining, and were excised for phosphorylation mass spectrometry analysis.

MS/MS spectra were searched by the Sequest algorithm against a database containing the mouse DGAT1 protein database and its reversed complement. Search parameters included a static modification of
57.02146 Da (carboxyamidomethylation) on Cys; dynamic modifications of $79.96633 \mathrm{Da}$ (phosphorylation) on Ser, Thr, and Tyr; and 15.99491 Da (oxidation) on Met. Results were first filtered to contain only fully tryptic peptides, and then other cutoffs were established to achieve maximum sensitivity levels at $0.1 \% \mathrm{FP}$ results using decoy matches as a guide. Sample-specific filters for the solution charge state and mass accuracy were used. A 3 Da mass tolerance window was applied separately to each analysis. After filtering by tryptic state, solution charge, and mass accuracy, only minimal filtering with Sequest scoring (XCorr and $\mathrm{dCn}$ ) values at the level of the entire dataset was then required to achieve less than $0.1 \% \mathrm{FP}$ rate.

\section{Bioinformatic analysis of DGAT1}

Cross-species sequence conservation was analyzed by ClustalX2 (Larkin et al. 2007). Potential phosphorylation sites of DGAT1 were predicted by NetPhosK (Blom et al. 2004) (http://www.cbs.dtu.dk/services/Net PhosK/) and PhosphoSitePlus (Hornbeck et al. 2012) (http://www.phosphosite.org/), and the parameters were set default.

\section{Statistics}

All experiment results were analyzed by GraphPad Prism 6.0 using one-way ANOVA.

Acknowledgments The authors acknowledge Prof. Mengqiu Dong and Dr. Li Tao for the help of phosphorylation mass spectrometry. This work was supported by the grants from the Ministry of Sciences and Technology of China (2011CBA00906; 2012DFG32160). 
Open Access This article is distributed under the terms of the Creative Commons Attribution 4.0 International License (http:// creativecommons.org/licenses/by/4.0/), which permits unrestricted use, distribution, and reproduction in any medium, provided you give appropriate credit to the original author(s) and the source, provide a link to the Creative Commons license, and indicate if changes were made.

\section{References}

Blom N, Sicheritz-Ponten T, Gupta R, Gammeltoft S, Brunak S (2004) Prediction of post-translational glycosylation and phosphorylation of proteins from the amino acid sequence. Proteomics 4:1633-1649

Cases S, Smith SJ, Zheng YW, Myers HM, Lear SR, Sande E, Novak S, Collins C, Welch CB, Lusis AJ, Erickson SK, Farese RV Jr (1998) Identification of a gene encoding an acyl CoA:diacylglycerol acyltransferase, a key enzyme in triacylglycerol synthesis. Proc Natl Acad Sci USA 95:13018-13023

Cases S, Stone SJ, Zhou P, Yen E, Tow B, Lardizabal KD, Voelker T, Farese RV Jr (2001) Cloning of DGAT2, a second mammalian diacylglycerol acyltransferase, and related family members. J Biol Chem 276:38870-38876

Chen HC, Smith SJ, Ladha Z, Jensen DR, Ferreira LD, Pulawa LK, McGuire JG, Pitas RE, Eckel RH, Farese RV Jr (2002) Increased insulin and leptin sensitivity in mice lacking acyl CoA:diacylglycerol acyltransferase 1. J Clin Invest 109:1049-1055

Chen HC, Rao M, Sajan MP, Standaert M, Kanoh Y, Miura A, Farese RV Jr, Farese RV (2004) Role of adipocyte-derived factors in enhancing insulin signaling in skeletal muscle and white adipose tissue of mice lacking Acyl CoA:diacylglycerol acyltransferase 1. Diabetes 53:1445-1451

Erion DM, Shulman GI (2010) Diacylglycerol-mediated insulin resistance. Nat Med 16:400-402

Goodpaster BH, He J, Watkins S, Kelley DE (2001) Skeletal muscle lipid content and insulin resistance: evidence for a paradox in endurance-trained athletes. J Clin Endocrinol Metab 86:5755-5761

Haagsman HP, de Haas CG, Geelen MJ, van Golde LM (1981) Regulation of triacylglycerol synthesis in the liver: a decrease in diacylglycerol acyltransferase activity after treatment of isolated rat hepatocytes with glucagon. Biochim Biophys Acta 664:74-81

Haagsman HP, de Haas CG, Geelen MJ, van Golde LM (1982) Regulation of triacylglycerol synthesis in the liver. Modulation of diacylglycerol acyltransferase activity in vitro. J Biol Chem 257:10593-10598

Han J (2011) Regulation of acyl-CoA: diacylglycerol acyltransferase- 1 by protein phosphorylation. Doctoral dissertation, University of Saskatchewan, Saskatoon

Han GH, Ye ML, Liu HW, Song CX, Sun DG, Wu YF, Jiang XN, Chen R, Wang CL, Wang LM, Zou HF (2010) Phosphoproteome analysis of human liver tissue by long-gradient nanoflow LC coupled with multiple stage MS analysis. Electrophoresis 31:1080-1089

Hornbeck PV, Kornhauser JM, Tkachev S, Zhang B, Skrzypek E, Murray B, Latham V, Sullivan M (2012) PhosphoSitePlus: a comprehensive resource for investigating the structure and function of experimentally determined post-translational modifications in man and mouse. Nucleic Acids Res 40:D261-D270

Humphrey SJ, Yang G, Yang P, Fazakerley DJ, Stockli J, Yang JY, James DE (2013) Dynamic adipocyte phosphoproteome reveals that Akt directly regulates mTORC2. Cell Metab 17:1009-1020
Huttlin EL, Jedrychowski MP, Elias JE, Goswami T, Rad R, Beausoleil SA, Villen J, Haas W, Sowa ME, Gygi SP (2010) A tissue-specific atlas of mouse protein phosphorylation and expression. Cell 143:1174-1189

Kelley DE, Goodpaster BH (2001) Skeletal muscle triglyceride. An aspect of regional adiposity and insulin resistance. Diabetes Care 24:933-941

Kelley DE, Goodpaster BH, Storlien L (2002) Muscle triglyceride and insulin resistance. Annu Rev Nutr 22:325-346

Kettenbach AN, Schweppe DK, Faherty BK, Pechenick D, Pletnev AA, Gerber SA (2011) Quantitative phosphoproteomics Identifies substrates and functional modules of aurora and pololike kinase activities in mitotic cells. Sci Signal 4:179

Larkin MA, Blackshields G, Brown NP, Chenna R, McGettigan PA, McWilliam H, Valentin F, Wallace IM, Wilm A, Lopez R, Thompson JD, Gibson TJ, Higgins DG (2007) Clustal W and Clustal X version 2.0. Bioinformatics 23:2947-2948

Liu L, Zhang Y, Chen N, Shi X, Tsang B, Yu Y-H (2007) Upregulation of myocellular DGAT1 augments triglyceride synthesis in skeletal muscle and protects against fat-induced insulin resistance. J Clin Investig 117:1679-1689

Machann J, Haring H, Schick F, Stumvoll M (2004) Intramyocellular lipids and insulin resistance. Diabetes Obes Metab 6:239-248

McFie PJ, Stone SL, Banman SL, Stone SJ (2010) Topological orientation of acyl-CoA:diacylglycerol acyltransferase-1 (DGAT1) and identification of a putative active site histidine and the role of the $\mathrm{n}$ terminus in dimer/tetramer formation. J Biol Chem 285:37377-37387

Moro C, Bajpeyi S, Smith SR (2008) Determinants of intramyocellular triglyceride turnover: implications for insulin sensitivity. Am J Physiol-Endoc M 294:E203-E213

Peng G, Li L, Liu Y, Pu J, Zhang S, Yu J, Zhao J, Liu P (2011) Oleate blocks palmitate-induced abnormal lipid distribution, endoplasmic reticulum expansion and stress, and insulin resistance in skeletal muscle. Endocrinology 152:2206-2218

Perseghin G, Scifo P, De Cobelli F, Pagliato E, Battezzati A, Arcelloni C, Vanzulli A, Testolin G, Pozza G, Del Maschio A, Luzi L (1999) Intramyocellular triglyceride content is a determinant of in vivo insulin resistance in humans - A H-1-C-13 nuclear magnetic resonance spectroscopy assessment in offspring of type 2 diabetic parents. Diabetes 48:1600-1606

Rodriguez MA, Dias C, Lau TE (1992) Reversible ATP-dependent inactivation of adipose diacylglycerol acyltransferase. Lipids 27:577-581

Russell AP, Gastaldi G, Bobbioni-Harsch E, Arboit P, Gobelet C, Deriaz O, Golay A, Witztum JL, Giacobino JP (2003) Lipid peroxidation in skeletal muscle of obese as compared to endurance-trained humans: a case of good vs. bad lipids? FEBS Lett 551:104-106

Samuel VT, Shulman GI (2012) Mechanisms for insulin resistance: common threads and missing links. Cell 148:852-871

Schenk S, Horowitz JF (2007) Acute exercise increases triglyceride synthesis in skeletal muscle and prevents fatty acid-induced insulin resistance. J Clin Invest 117:1690-1698

Shulman GI, Rothman DL, Jue T, Stein P, DeFronzo RA, Shulman RG (1990) Quantitation of muscle glycogen synthesis in normal subjects and subjects with non-insulin-dependent diabetes by $13 \mathrm{C}$ nuclear magnetic resonance spectroscopy. New Engl J Med 322:223-228

Siloto RM, Madhavji M, Wiehler WB, Burton TL, Boora PS, Laroche A, Weselake RJ (2008) An N-terminal fragment of mouse DGAT1 binds different acyl-CoAs with varying affinity. Biochem Biophys Res Commun 373:350-354

Trinidad JC, Barkan DT, Gulledge BF, Thalhammer A, Sali A, Schoepfer R, Burlingame AL (2012) Global Identification and 
Characterization of Both O-GlcNAcylation and Phosphorylation at the Murine Synapse. Mol Cell Proteomics 11:215-229

Villen J, Beausoleil SA, Gerber SA, Gygi SP (2007) Large-scale phosphorylation analysis of mouse liver. Proc Natl Acad Sci USA 104:1488-1493

Weselake RJ, Madhavji M, Szarka SJ, Patterson NA, Wiehler WB, Nykiforuk CL, Burton TL, Boora PS, Mosimann SC, Foroud NA, Thibault BJ, Moloney MM, Laroche A, Furukawa-Stoffer TL (2006) Acyl-CoA-binding and self-associating properties of a recombinant $13.3 \mathrm{kDa} \mathrm{N}$-terminal fragment of diacylglycerol acyltransferase-1 from oilseed rape. BMC Biochem 7:24

Wilson-Grady JT, Haas W, Gygi SP (2013) Quantitative comparison of the fasted and re-fed mouse liver phosphoproteomes using lower $\mathrm{pH}$ reductive dimethylation. Methods 61:277-286
Xu Y, Wang L, He J, Bi Y, Li M, Wang T, Wang L, Jiang Y, Dai M, Lu J, Xu M, Li Y, Hu N, Li J, Mi S, Chen CS, Li G, Mu Y, Zhao J, Kong L, Chen J, Lai S, Wang W, Zhao W, Ning G, China Noncommunicable Disease Surveillance Group (2013) Prevalence and control of diabetes in Chinese adults. JAMA 310:948-959

Yen C-LE, Stone SJ, Koliwad S, Harris C, Farese RV Jr (2008) Thematic Review Series: glycerolipids. DGAT enzymes and triacylglycerol biosynthesis. J Lipid Res 49:2283-2301

Yu YH, Zhang Y, Oelkers P, Sturley SL, Rader DJ, Ginsberg HN (2002) Posttranscriptional control of the expression and function of diacylglycerol acyltransferase-1 in mouse adipocytes. J Biol Chem 277:50876-50884 